\title{
Investigation for Loss Generation Mechanisms of Flow in Turbomachinery by Using Curved Square Duct* (3rd Report, Influence of Curved Angle)
}

\author{
Hoshio TSUJITA**, Shimpei MIZUKI'** and Atsumasa YAMAMOTO***
}

\begin{abstract}
In a series of present studies, the flows within the stationary curved ducts are analyzed numerically with the aerodynamic or the geometrical parameters affecting the loss generation caused by the passage vortex within a passage of a turbomachinery. In the former reports, the inlet boundary layer thickness and the inlet velocity distortion were taken as the aerodynamic parameter and the aspect ratio of the cross-section was chosen as the geometrical parameter. In this report, the effects of the curved angle of the bend on the loss generation caused by the passage vortex are examined by relating the bend of curved duct to the blade-to-blade surface of an axial flow turbine cascade. The curved angle is changed from 90 to 160 degrees by 10 degrees with fixing the length of arc for the mean radius of curvature of the bend or with fixing the mean radius of curvature.
\end{abstract}

Key Words: Turbomachinery, Secondary Flow, Numerical Analysis, Loss Generation, Passage Vortex, Curved Duct, Curved Angle

\section{Introduction}

The secondary flows affecting the loss generation within the cascade of a turbomachinery are the horseshoe vortex, the passage vortex, the tip leakage vortex and so on. In these secondary flows, the passage vortex generates the major part of the losses in the flow channels. The curved ducts are considered to be fundamental models for the generation of the passage vortex. The objective of this study is to reveal the effects of the passage vortex on the loss generation mechanisms by numerically analyzing the flow within the stationary curved duct for various aerodynamic and geometrical parameters, which affect the behavior of passage vortex within the passage of the practical turbomachine. Because the configuration of the curved duct is much simpler than that of the cascade of turbomachines, the rectangular computational grids can be easily applied to the entire computational domain. Therefore,

* Received 1st March, 2006 (No. 04-0853). Japanese Original: Trans. Jpn. Soc. Mech. Eng., Vol.71, No.705, B (2005), pp.1231-1238 (Received 22nd July, 2004)

** Department of Mechanical Engineering, Hosei University, 3-7-2 Kajino-cho, Koganei-shi, Tokyo 184-8584, Japan. E-mail: tsujita@k.hosei.ac.jp

*** Aerospace Research Center, Japan Aerospace Exploration Agency, 7-44-1 Jindaiji-higashi-machi, Chofu-shi, Tokyo 182-8522, Japan the computed results for the curved duct are considered to possess the high resolutions. The effects of Mach number and the shock/boundary layer interaction caused by compressibility, which would have predominant effects on the practical turbine cascade, are excluded here in order to focus on the effects of passage vortex on the loss generation. Thus, the flows analyzed in this study are at relatively low Reynolds number and low Mach number compared to those in the practical turbomachines. In the first report ${ }^{(1)}$, the accuracy of the present computational code was verified by the comparison of the computed results with the experimental data. In addition, the effects of the inlet velocity profile as the aerodynamic parameter on the formation of passage vortex and the associated loss generation were examined in detail. The effects of the cross-sectional aspect ratio of the curved ducts as the geometrical parameter on them were clarified in the second report ${ }^{(2)}$.

On the other hand, the several investigations ${ }^{(3)-(5)}$ concerning the increase of blade loading for turbine cascades have been made in order to improve the performance characteristics of gas turbines. However, the increase of the turbine loading requires the increase of the blade turning angle. Hence, the pressure gradient in the pitchwise direction becomes steeper and, as a result, the secondary flow including the passage vortex, which reduces the turbine efficiency, becomes strong. Therefore, it is important 
to investigate the effects of the turning angle on the formation of passage vortex and the associated loss generation in order to attain the increase of blade loading without the reduction of turbine performance.

In this study, the bend of curved duct was related to the blade-to-blade surface of an axial flow turbine cascade as shown in Fig. 1. Then, the numerical analyses of flow within the curved ducts were carried out with the variation of the curved angle which corresponded to the turning angle of turbine blade, in order to clarify the effects of the curved angle on the formation of passage vortex and the associated loss generation.

\section{Nomenclature}

$A_{\text {cps }}$ : loading coefficient

$C_{p s}:$ static pressure coefficient $=\left(p-p_{r e f}\right) /\left(\rho V_{c}^{2} / 2\right)$

$C_{p t}$ : total pressure loss coefficient $=\left(p_{\text {tref }}-p_{t}\right) /\left(\rho V_{c}^{2} / 2\right)$

$D$ : hydraulic diameter

$H$ : duct height

$L_{c}:$ length of bend $=2 R_{c} \pi \alpha / 360$

$L_{R}$ : geometrical reference length $(=0.04 \mathrm{~m})$

$p:$ static pressure

$p_{\text {ref }}$ : static pressure at $\theta=0 \mathrm{deg}$. and $r^{*}=z^{*}=0$

$p_{t}:$ total pressure

$p_{\text {tref }}:$ mass-averaged total pressure at inlet of duct

$Q$ : volumetric flow rate

$R_{a}:$ cross-sectional aspect ratio $=S / H$

$R_{c}$ : mean radius of curvature $=0.5\left(r_{i}+r_{o}\right)$

$R_{e}:$ Reynolds number $=\rho D V_{c} / \mu$

$r_{i}$ : radius of curvature of inner wall

$r_{o}$ : radius of curvature of outer wall

$S:$ duct width

$V_{c}$ : bulk mean velocity

$X_{h}$ : streamwise distance normalized by $L_{R}$ with origin at $\theta=0 \mathrm{deg}$.

$X_{D}$ : streamwise distance normalized by $L_{R}$ with origin at $\theta=\alpha$

$z$ : spanwise coordinate

$z^{*}:$ normalized spanwise coordinate $=z /(S / 2)$

$\Delta C_{p t, i}$ : total pressure loss coefficient caused by passage vortex

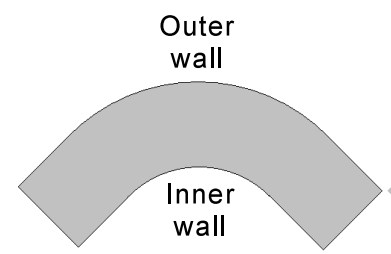

(a) Curved duct

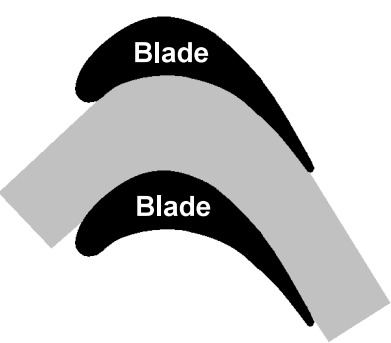

(b) Axial flow turbine cascade
Fig. 1 Correspondence of curved duct to axial flow turbine cascade
$\Delta \theta_{y}:$ flow deflection angle at exit of bend $=\theta_{y}-\alpha$

$\alpha$ : curved angle

$\rho:$ density

$\theta:$ streamwise coordinate within bend

$\theta_{y}:$ flow angle at exit of bend $(\theta=\alpha)$

$\omega_{\text {iabs }}$ : absolute value of streamwise component of vorticity

Subscripts

,$i$ : mass-averaged value on the cross-section

, $i j$ : mass-averaged value in duct height $H(k)$ direction

, $i k$ : mass-averaged value in duct width $S(j)$ direction

\section{Computational Method}

The momentum equations for the three-dimensional steady incompressible turbulent flow are solved in the physical component tensor form with the standard $k-\varepsilon$ turbulence model. The wall function method is used for the wall boundary condition. The finite volume method is applied for the discretization of governing equations. The convection term is estimated by the third order upwind difference scheme. The solution method is the SIMPLE algorithm. The detail of the computational method is shown in Refs. (6) and (7). The verification for the accuracy of the present computational code was carried out in the first report $^{(1)}$ by comparing with the experimental data.

\section{Configuration of Curved Duct}

The configuration of curved duct is shown in Fig. 2. The fundamental model of this curved duct has the curved angle $\alpha=90$ degrees, the mean radius of curvature $R_{c}=$ $0.092 \mathrm{~m}$ and the length of bend $L_{c}=0.145 \mathrm{~m}$. The definition of $L_{c}$ is given in Fig. 3. In the second report ${ }^{(2)}$ concerning the effects of cross-sectional aspect ratio $R_{a}$ (=S/H), it was clarified that the loss caused by the passage vortex was extremely increased by the decrease of $R_{a}$ lower than 2.0. Thus, in this study, the aspect ratio $R_{a}$ of curved duct was set to 1.0 and 3.0. Then, the duct height

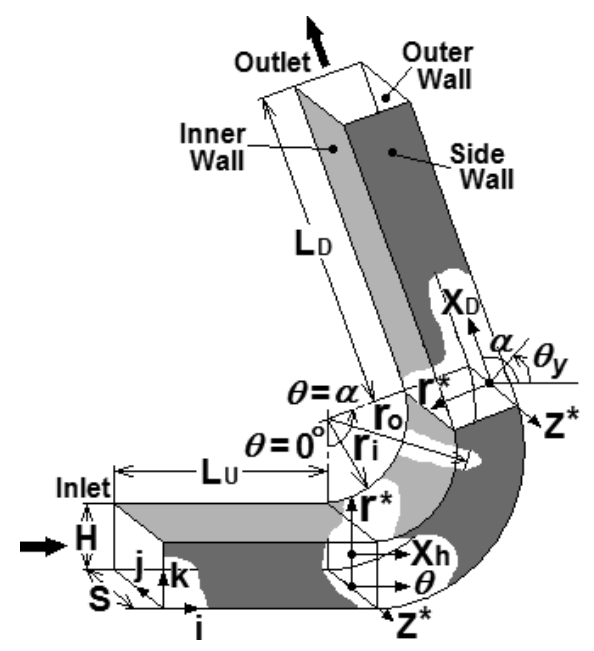

Fig. 2 Curved duct 


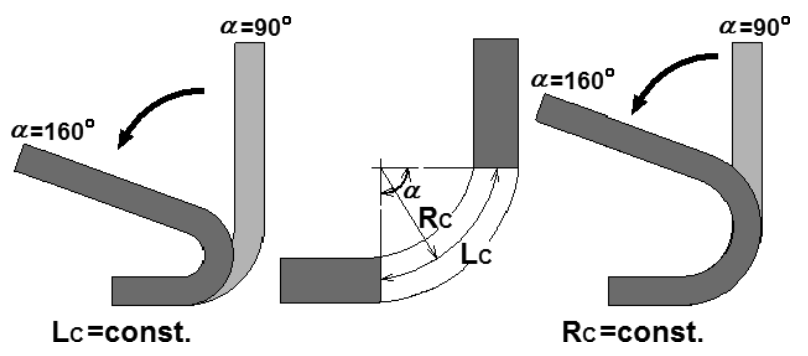

Fig. 3 Variation of curved angle

Table 1 Parameters depending on $R_{a}$

\begin{tabular}{|c|c|c|c|c|}
\hline$R_{a}$ & $H_{(m)}$ & $D_{(m)}$ & $R_{e}$ & $Q_{\left(m^{3} / s\right)}$ \\
\hline 1.0 & 0.040 & 0.040 & 40000 & 1.60 \\
3.0 & 0.023 & 0.035 & 34263 & $\times 10^{-3}$ \\
\hline
\end{tabular}

Table 2 Geometrical parameters

\begin{tabular}{|c|c|c|c|c|}
\hline \multirow{2}{*}{$\alpha$ (deg.) } & \multicolumn{2}{|c|}{$L_{c}=$ const. } & \multicolumn{2}{|c|}{$R_{C}=$ const. } \\
\hline & $L_{c}(\mathrm{~m})$ & $R_{c}(\mathrm{~m})$ & $L_{c}(\mathrm{~m})$ & $R_{c}(\mathrm{~m})$ \\
\hline 90 & \multirow{8}{*}{0.145} & 0.092 & 0.145 & \multirow{8}{*}{0.092} \\
\hline 100 & & 0.0828 & 0.161 & \\
\hline 110 & & 0.0753 & 0.177 & \\
\hline 120 & & 0.0690 & 0.193 & \\
\hline 130 & & 0.0637 & 0.209 & \\
\hline 140 & & 0.0591 & 0.225 & \\
\hline 150 & & 0.0552 & 0.241 & \\
\hline 160 & & 0.0518 & 0.257 & \\
\hline
\end{tabular}

$H$ and the duct width $S$ for these two aspect ratios were decided to make the same cross-sectional area with each other in order to fix the volumetric flow rate $Q$ (Table 1). Furthermore, for these two curved ducts with the different $R_{a}$, the curved angle $\alpha$ was varied from 90 degrees to 160 degrees with the interval of 10 degrees by two kinds of different manners illustrated in Fig. 3. In the first manner, the $\alpha$ was varied with fixing $L_{c}$, and in the second one, it was varied with fixing $R_{c}$ as shown in Table 2. These two manners will be referred as the case of $L_{c}=$ const. and the case of $R_{c}=$ const. in the following, respectively. The computations were performed for 30 kinds of curved ducts described in Tables 1 and 2. The increase of curved angle $\alpha$ results in the increase of the force acting on the bend of curved duct, which corresponds to the blade loading of turbine cascade. In general, the increase of $\alpha$ with fixing $L_{c}$ restraints the increase of the friction loss on the solid walls but increases the strength of passage vortex and the associated loss generation due to the decrease of $R_{c}$. On the other hand, the increase of $\alpha$ with fixing $R_{c}$ restraints the loss caused by the passage vortex but increases the friction loss due to the increase of solid wall surface area.

\section{Results and Discussion}

The streamwise velocity of $1.0 \mathrm{~m} / \mathrm{s}$ was given uni-

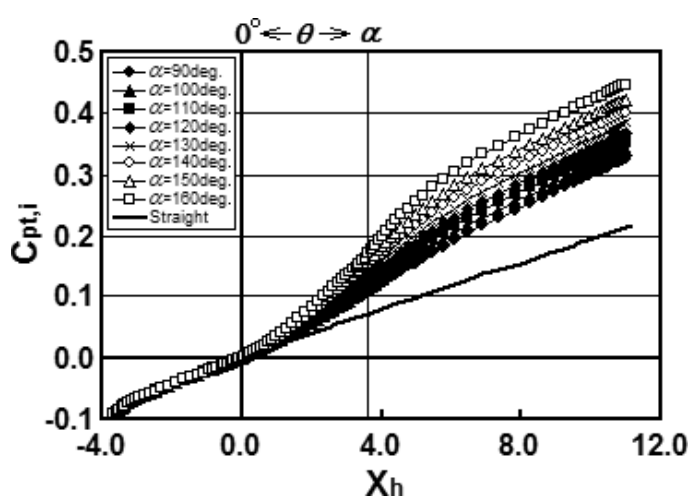

Fig. 4 Total pressure loss coefficient $\left(L_{c}=\right.$ const. $)$

formly at the inlet boundary cross section for every curved duct in Table 2. The volumetric flow rate $Q$ was fixed to $1.6 \times 10^{-3} \mathrm{~m}^{3} / \mathrm{s}$ in this study, because the cross-sectional area was fixed to constant irrespective of the aspect ratio $R_{a}$ as mentioned before. The every curved duct has the straight ducts of $L_{U}=3.75 L_{R}$ and $L_{D}=7.5 L_{R}$ upstream and downstream of the bend, respectively (Fig. 2), where $L_{R}$ is the reference length and equal to the hydraulic diameter $D(=0.04 \mathrm{~m})$ of the duct with $R_{a}=1.0$. The numbers of the computational grid on the cross section are 35 (in duct height direction) $\times 35$ (in duct width direction) for the duct with $R_{a}=1.0$ and 27 (in duct height direction) $\times 55$ (in duct width direction) for that with $R_{a}=3.0$. The number in the streamwise direction is 95 in the case of $L_{c}=$ const. and increased up to 115 according to the increase of $L_{c}$ in the case of $R_{c}=$ const.

\section{1 Case of $L_{c}=$ const. for duct with $R_{a}=1.0$}

The computations for the duct with $R_{a}=1.0$ were performed for each curved angle $\alpha$ in the case of $L_{c}=$ const. shown in Table 2. In this case, the increase of $\alpha$ reduces the value of $R_{c}$.

Figure 4 shows the streamwise distributions of the mass averaged total pressure loss coefficient $C_{p t, i}$. The coordinate $X_{h}$ is the streamwise distance normalized by $L_{R}$ with the origin at $\theta=0 \mathrm{deg}$. (Fig. 2). The reference point for the distributions in Fig. 4 is taken at the inlet of the bend $\left(X_{h}=0.0\right)$. The flow within the straight duct, which had the same configuration for the cross section as that of the curved duct, was analyzed with the same inlet boundary conditions as those for the curved duct. The $C_{p t, i}$ distribution for the straight duct is also shown by the solid line in Fig. 4. The $C_{p t, i}$ of the straight duct exhibits the loss caused by the development of the boundary layer on the solid walls without the curvature. The results indicate that the increase of $\alpha$ increases the total pressure loss $C_{p t, i}$. The loss caused by the passage vortex $\Delta C_{p t, i}$ is defined as the difference between the $C_{p t, i}$ of the straight duct and that of the curved duct in the present study. The streamwise distribution of $\Delta C_{p t, i}$ is shown in Fig. 5 for each $\alpha$. The $\Delta C_{p t, i}$ is increased from the inlet of the bend by the 


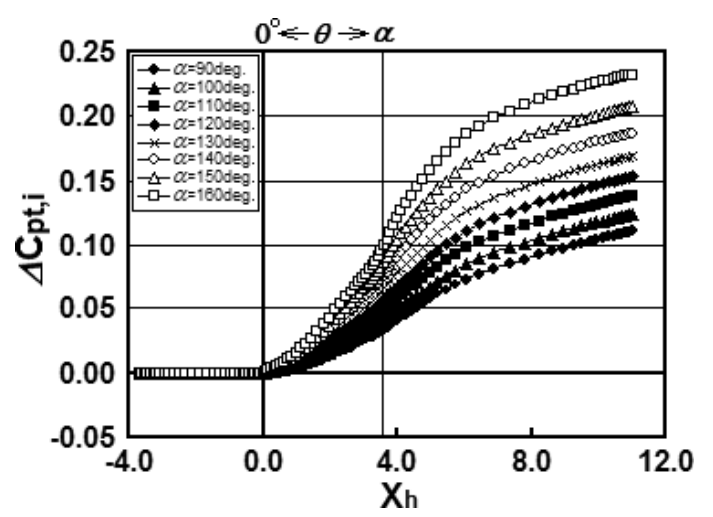

Fig. 5 Total pressure loss by passage vortex $\left(L_{c}=\right.$ const. $)$

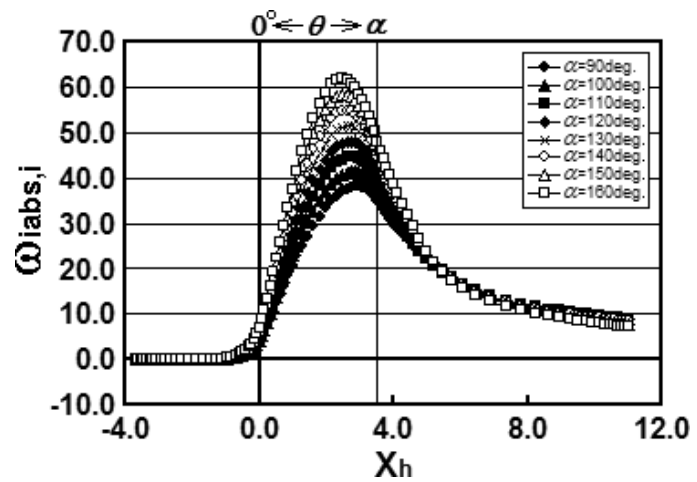

Fig. 6 Absolute value of streamwise component of vorticity ( $L_{c}=$ const. $)$

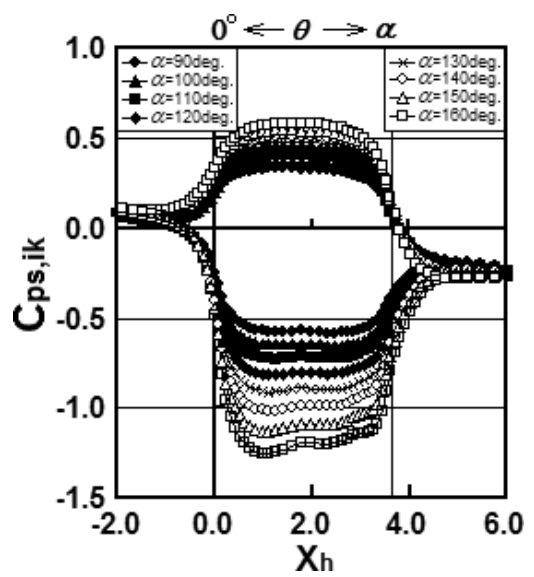

Fig. 7 Static pressure coefficient on inner and outer walls ( $L_{c}=$ const.)

increase of $\alpha$.

The streamwise distributions of the absolute value of streamwise component of vorticity mass-averaged on the cross-section $\omega_{i a b s, i}$, which amounts to the intensity of the secondary flow, are shown in Fig. 6. Figure 7 gives the streamwise distributions of the static pressure coefficient mass-averaged in $j$-direction $C_{p s, i k}$ on the inner and the outer walls within the bend. They are considered to correspond to the blade loading distribution of a turbine cascade. The $\omega_{i a b s, i}$ increases rapidly from the inlet of the

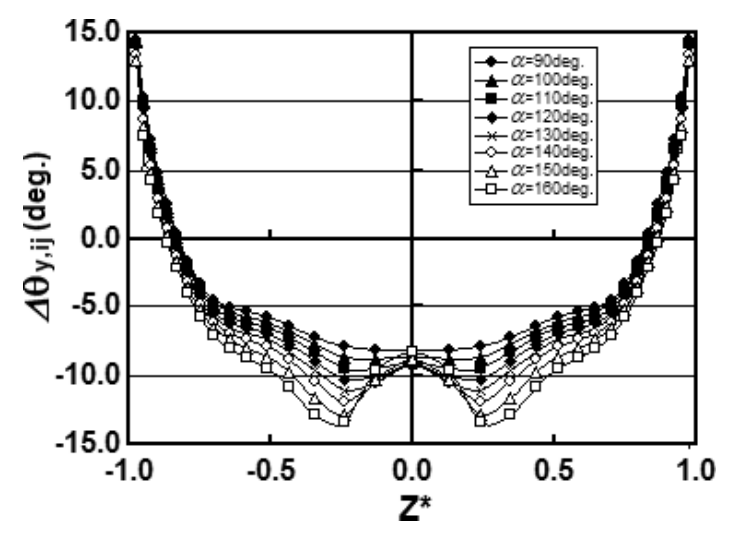

Fig. 8 Flow angle at $\theta=\alpha\left(L_{c}=\right.$ const. $)$

bend for every $\alpha$ due to the generation and the development of passage vortex. The $\Delta C_{p t, i}$ also increases within the bend simultaneously (Fig. 5). This phenomenon is considered to be attributed to the increase of friction loss caused by the development of passage vortex, because the passage vortex has the high secondary flow velocity component particularly near the solid walls. Near the exit of the bend, the $\omega_{i a b s, i}$ decreases rapidly due to the decline of the passage vortex. However, the $\Delta C_{p t, i}$ continues to increase. This increase of $\Delta C_{p t, i}$ downstream of the bend is attributed to the increase of the mixing loss caused by the decline of passage vortex ${ }^{(8)}$ as clearly recognized by the comparison between the increasing rate of $\Delta C_{p t, i}$ and the decreasing rate of $\omega_{i a b s, i}$ downstream of the bend (Figs. 5 and 6). The increase of $\alpha$ increases the $\omega_{i a b s, i}$ as seen in Fig. 6. This phenomenon can be explained by the fact that the increase of $\alpha$ enlarges the difference between the static pressure on the inner wall and that on the outer wall (Fig. 7), and consequently strengthens the passage vortex. Therefore, the increase of $\alpha$ raises the $\Delta C_{p t, i}$ by the increases of the friction loss within the bend and of the mixing loss from the exit of the bend. The former is caused by the development of passage vortex and the latter is by the decline of passage vortex.

Figure 8 shows the distribution in $z^{*}$-direction of $\Delta \theta_{y, i j}$ which is the $\Delta \theta_{y}$ mass-averaged in $k$-direction at the exit of the bend $(\theta=\alpha)$. The $\Delta \theta_{y}\left(=\theta_{y}-\alpha\right)$ is defined as the deflection of the bend exit flow angle $\theta_{y}$ from the bend curved angle $\alpha$. The definition of $\theta_{y}$ is shown in Fig. 2 . The $\Delta \theta_{y, i j}$ is considered to be related to the turbine cascade exit flow angle which influences the work of the axial flow turbine. The $z^{*}$ is the spanwise coordinate normalized by $S / 2$ (Fig. 2). The increase of $\alpha$ decreases the $\Delta \theta_{y, i j}$ particularly around the middle $\left(z^{*}=0.0\right)$ of the duct width and enhances the tendency toward under-turn. However, in the vicinity of $z^{*}=0.0$, the $\Delta \theta_{y, i j}$, is increased locally by the increase of $\alpha$. The cause of this behavior will be discussed in the following.

\subsection{Case of $\boldsymbol{R}_{c}=$ const. for duct with $\boldsymbol{R}_{a}=\mathbf{1 . 0}$}

The flow within the duct with $R_{a}=1.0$ was computed 
for each curved angle $\alpha$ in the case of $R_{c}=$ const. as shown in Table 2. In this case, the increase of $\alpha$ results in the increase of $L_{c}$.

The streamwise distributions of $C_{p t, i}$ for each $\alpha$ are shown in Fig. 9, in which the solid line is that for the straight duct with the same configuration for the cross section as that of the curved duct. Figures 10 and 11 give the streamwise distributions of $\Delta C_{p t, i}$ and $\omega_{i a b s, i}$, respectively. Figure 12 shows the streamwise distributions of the static pressure $C_{p s, i k}$ on the inner and the outer walls within the bend. Although the difference between the $C_{p t, i}$ distributions for the curved ducts and that for the straight duct appears gradually from the inlet of the bend $\left(X_{h}=0.0\right)$, the

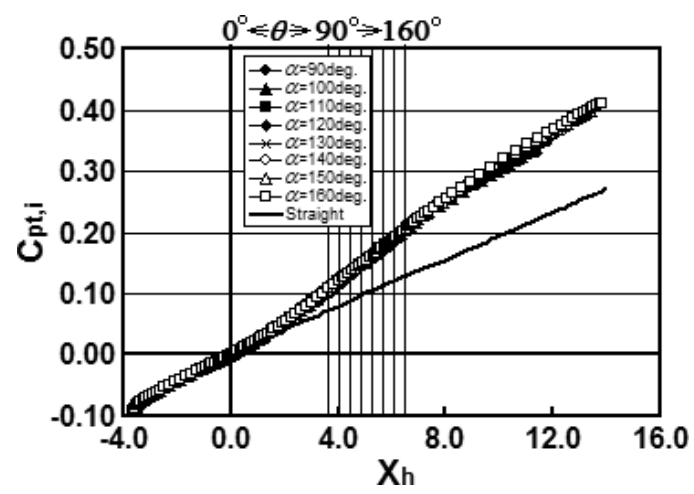

Fig. 9 Total pressure loss coefficient $\left(R_{c}=\right.$ const. $)$

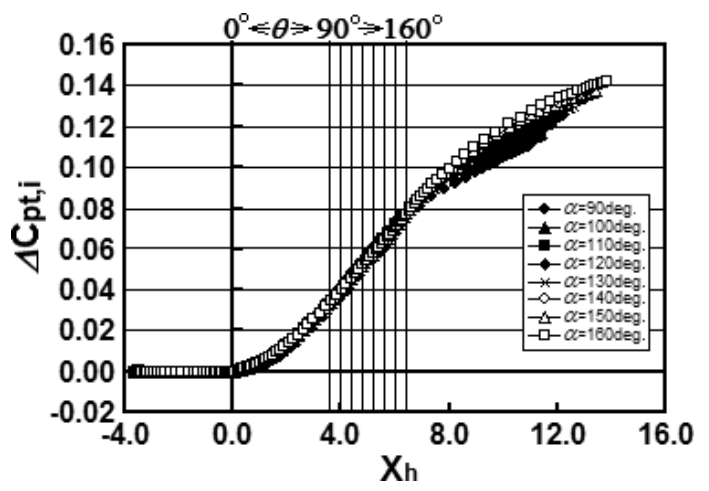

Fig. 10 Total pressure loss by passage vortex $\left(R_{c}=\right.$ const. $)$

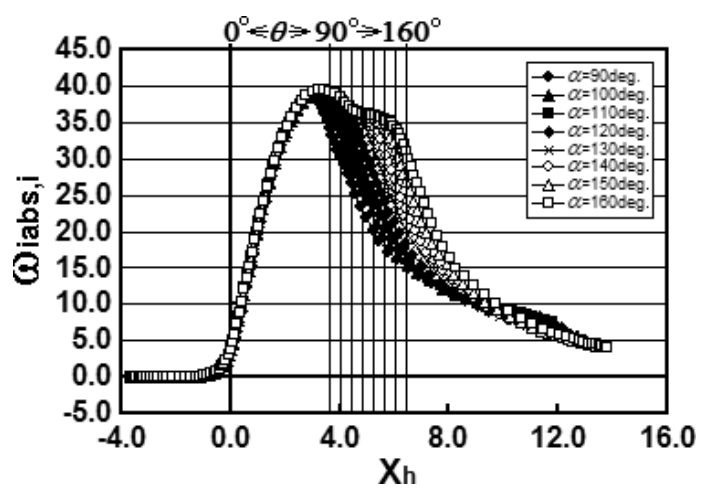

Fig. 11 Absolute value of streamwise component of vorticity $\left(R_{c}=\right.$ const. $)$ effect of the increase of $\alpha$ on the difference between those for the curved ducts is very little at the same position of $X_{h}$ (Fig. 9). The similar tendency is clearly recognized also in the effect of $\alpha$ on the $\Delta C_{p t, i}$ distribution (Fig. 10). It is apparent from Fig. 11 that the maximum value of $\omega_{i a b s, i}$ which amounts to the strength of passage vortex is not affected by the increase of $\alpha$. This means that the maximum difference between the static pressures on the inner and the outer walls is independent of $\alpha$ as seen in Fig. 12. As a result, the $\Delta C_{p t, i}$ is hardly affected by the increase of $\alpha$ in the case of $R_{c}=$ const. By comparing Fig. 11 with Fig. 12, it is observed that the strength of passage vortex is almost preserved up to the exit of the bend for each $\alpha$, because the difference between the static pressures on the inner and the outer walls is nearly constant within the bend.

Figure 13 shows the distribution in $z^{*}$-direction of $\Delta \theta_{y, i j}$ at the exit of the bend $(\theta=\alpha)$. The influence of the increase of $\alpha$ on the distribution of $\Delta \theta_{y, i j}$ becomes weak compared with that in the case of $L_{c}=$ const. (Fig. 8). Thus, the strength of passage vortex is not affected by the increase of $\alpha$ as mentioned before. However, the increase of $\Delta \theta_{y, i j}$ in the vicinity of $z^{*}=0.0$ by the increase of $\alpha$ also appears as observed in the case of $L_{c}=$ const. Figure 14 shows the secondary flow velocity vectors and the secondary flow streamlines in the half domain on the

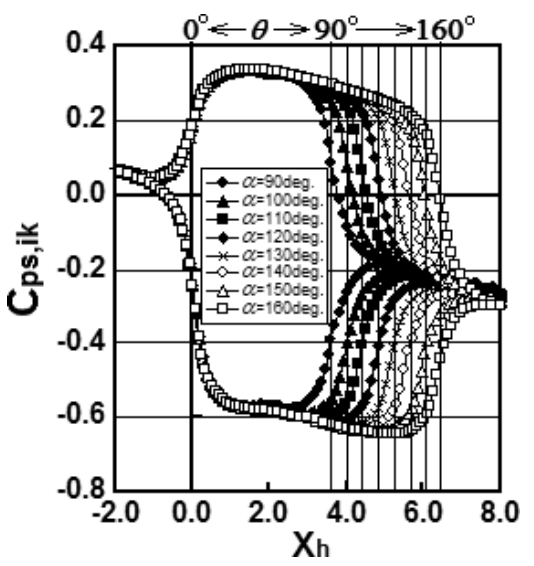

Fig. 12 Static pressure coefficient on inner and outer walls ( $R_{c}=$ const. $)$

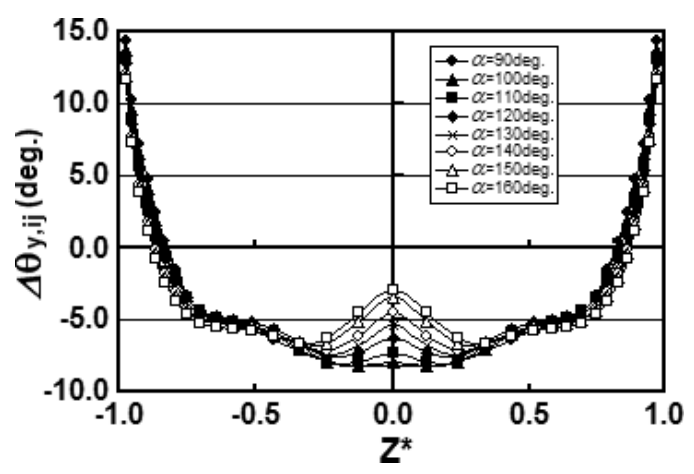

Fig. 13 Flow angle at $\theta=\alpha\left(R_{c}=\right.$ const. $)$ 


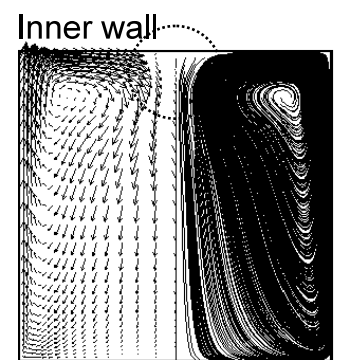

(a) $\alpha=90 \mathrm{deg}$.

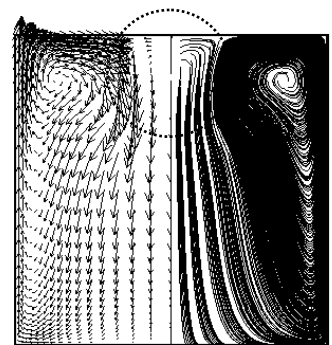

(b) $\alpha=160 \mathrm{deg}$. ( $L_{c}=$ const.)

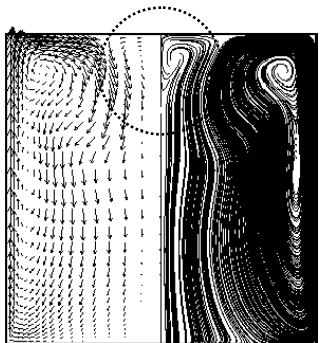

(c) $\alpha=160 \mathrm{deg}$. ( $R_{c}=$ const. $)$

Fig. 14 Secondary flow velocity vectors and streamlines at $\theta=\alpha$

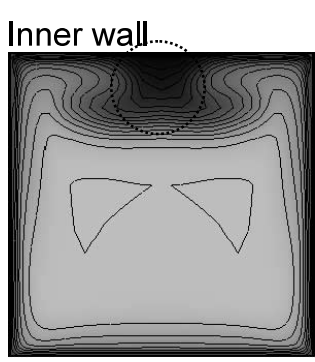

(a) $\alpha=90 \mathrm{deg}$.

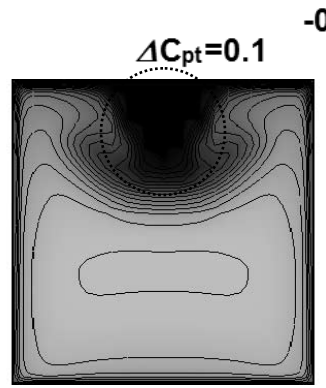

(b) $\alpha=160 \mathrm{deg}$.

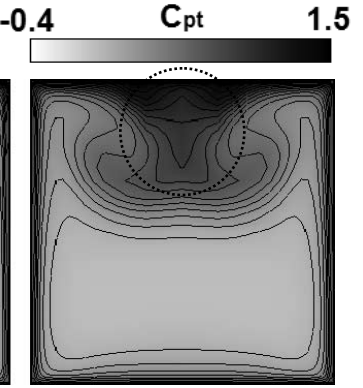

(c) $\alpha=160 \mathrm{deg}$. ( $R_{c}=$ const.)

Fig. 15 Total pressure loss distribution at $\theta=\alpha$

cross section at the exit of the bend $(\theta=\alpha)$ for the curved ducts of $\alpha=90$ degrees, $\alpha=160$ degrees in the case of $L_{c}=$ const. and $\alpha=160$ degrees in the case of $R_{c}=$ const. Figure 15 shows the total pressure loss $C_{p t}$ distributions on the same cross sections as those in Fig. 14. In these figures, the increases of $\alpha$ in both of the cases of $L_{c}=$ const. and $R_{c}=$ const. enlarge the high loss region in the middle of the inner wall (enclosed by the dotted line). As a result, a pair of passage vortex is separated by the enlargement of high loss region, where a pair of small vortex with the opposite rotation to that of a pair of passage vortex appears, clearly for $\alpha=160$ degrees in the case of $R_{c}=$ const. (Fig. 14 (c)) and, slightly for that in the case of $L_{c}=$ const. (Fig. 14 (b)). Therefore, the increase of $\Delta \theta_{y, i j}$ in the vicinity of $z^{*}=0.0$ by the increase $\alpha$ observed in Figs. 8 and 13 is caused by the flow toward the inner wall, which constitutes a pair of small vortex within the high loss region.

\subsection{Comparison between cases of $L_{c}=$ const. and $R_{c}=$ const. for duct with $\boldsymbol{R}_{a}=\mathbf{1 . 0}$}

Figure 16 shows the relationship between the loading coefficient $A_{c p s}$ and the curved angle $\alpha$. The coefficient $A_{c p s}$ is defined as the area surrounded by the two curves of $C_{p s, i k}$ on the inner and the outer walls shown in Figs. 7 ( $L_{c}=$ const.) and $12\left(R_{c}=\right.$ const. $)$. The $A_{c p s}$, which corresponds to the blade loading of turbine cascade, is increased by the increase of $\alpha$. Moreover, the increase of $\alpha$ makes the $A_{c p s}$ in the case of $L_{c}=$ const. higher than that in the case of $R_{c}=$ const.

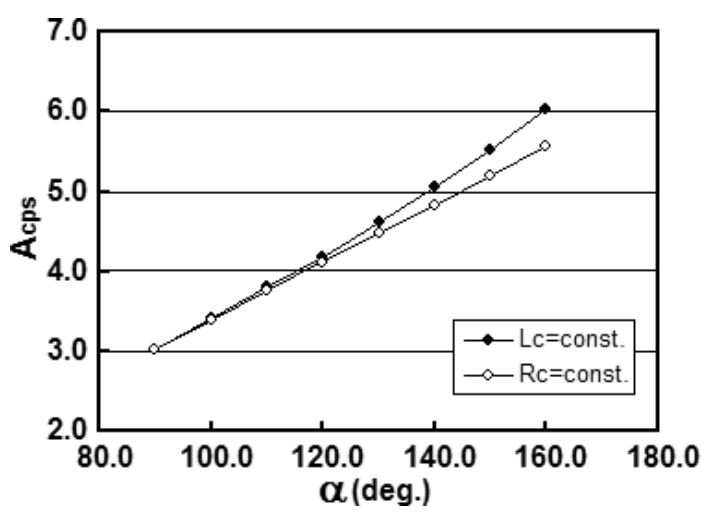

Fig. 16 Influence of $\alpha$ on loading coefficient $A_{c p s}\left(R_{a}=1.0\right)$

The influences of $\alpha$ on the $\Delta C_{p t, i}$ and the $C_{p t, i}$ at exit of the bend $(\theta=\alpha)$ in the cases of $L_{c}=$ const. and $R_{c}=$ const. are shown in Figs. 17 and 18, respectively. By the increase of $\alpha$, the loss caused by the passage vortex $\Delta C_{p t, i}$ in the case of $L_{c}=$ const. becomes higher than that in the case of $R_{c}=$ const. (Fig. 17), because the passage vortex within the bend in the former case is stronger than that in the latter as seen in Figs. 6 and 11. On the other hand, the total pressure loss $C_{p t, i}$ shows the higher value for the case of $R_{c}=$ const. as the $\alpha$ increases (Fig. 18). This is caused by the higher friction loss on the solid walls in the case of $R_{c}=$ const., since the length of the bend $L_{c}$ in the case of $R_{c}=$ const. is increased by the increase of $\alpha$ (Table 2).

Figures 19 and 20 show the influences of $\alpha$ on the 


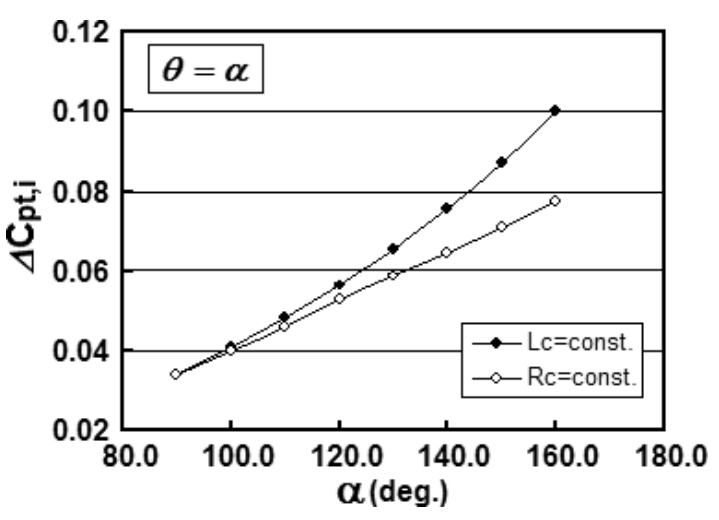

Fig. 17 Influence of $\alpha$ on $\Delta C_{p t, i}$ at $\theta=\alpha\left(R_{a}=1.0\right)$

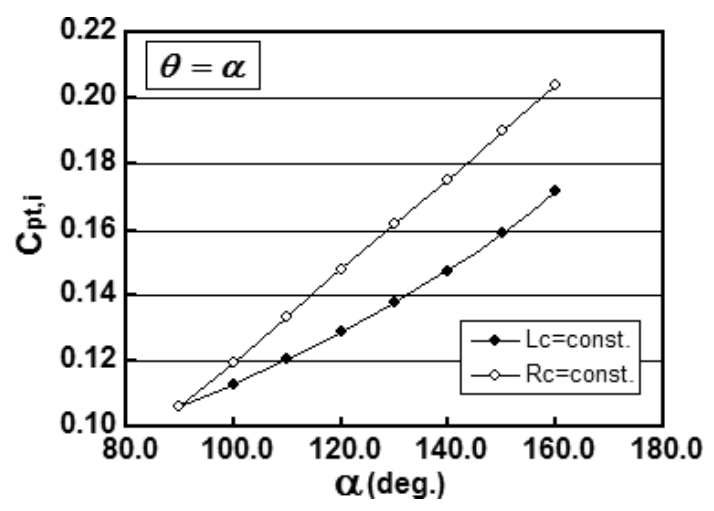

Fig. 18 Influence of $\alpha$ on $C_{p t, i}$ at $\theta=\alpha\left(R_{a}=1.0\right)$

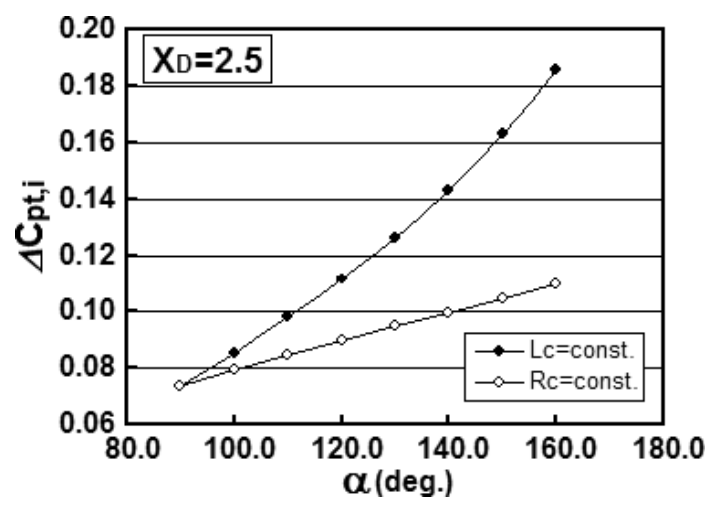

Fig. 19 Influence of $\alpha$ on $\Delta C_{p t, i}$ at $X_{D}=2.5\left(R_{a}=1.0\right)$

$\Delta C_{p t, i}$ and the $C_{p t, i}$ at $X_{D}=2.5$ downstream of the bend in the cases of $L_{c}=$ const. and $R_{C}=$ const., respectively, where $X_{D}$ is the streamwise distance normalized by $L_{R}$ with the origin at the exit of the bend (Fig. 2). By the increase of $\alpha$, the $\Delta C_{p t, i}$ in the case of $L_{c}=$ const. becomes higher than that in the case of $R_{c}=$ const. (Fig. 19) as at the exit of the bend (Fig. 17). However, the $C_{p t, i}$ shows the higher value for the case of $L_{c}=$ const. (Fig. 20). This behavior is different from that at the exit of the bend (Fig. 18) and is attributed to the fact that the increasing rate of $\Delta C_{p t, i}$ from the exit of the bend $(\theta=\alpha)$ up to $X_{D}=2.5$ becomes much higher for the case of $L_{c}=$ const. by the increase of $\alpha$, as recognized in the comparison between Figs. 17 and

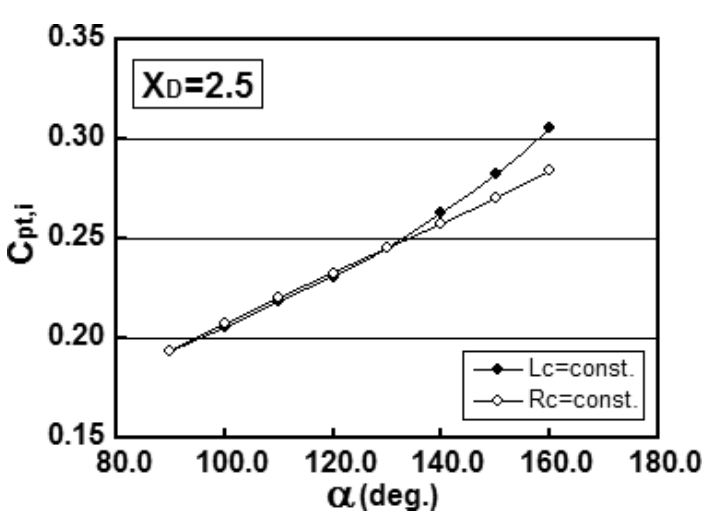

Fig. 20 Influence of $\alpha$ on $C_{p t, i}$ at $X_{D}=2.5\left(R_{a}=1.0\right)$

19. This means that the loss caused by the passage vortex is predominant in the loss generation downstream of the bend. Therefore, for the duct with $R_{a}=1.0$, the increase of $\alpha$ in the case of $L_{c}=$ const. increases not only the $A_{c p s}$ but also the $C_{p t, i}$ more than in the case of $R_{c}=$ const.

\subsection{Comparison between ducts with $R_{a}=1.0$ and} $\boldsymbol{R}_{a}=\mathbf{3 . 0}$

The computations for the flows within the duct with $R_{a}=3.0$ were performed by varying the curved angle $\alpha$ in the same manner as for the duct with $R_{a}=1.0$. The results showed the similar effects of $\alpha$ on the total pressure loss $C_{p t, i}$, the loss caused by passage vortex $\Delta C_{p t, i}$, the streamwise component of vorticity $\omega_{i a b s, i}$ and the static pressure $C_{p s, i k}$ on the inner and the outer wall within the bend to those for the duct with $R_{a}=1.0$. Figure 21 shows the distributions in $z^{*}$-direction of $\Delta \theta_{y, i j}$ at the exit of the bend $(\theta=\alpha)$ in the cases of $L_{c}=$ const. and $R_{c}=$ const. The increase of $\alpha$ decreases $\Delta \theta_{y, i j}$ particularly around the middle $\left(z^{*}=0.0\right)$ of the duct width in the case of $L_{c}=$ const., but it hardly affects on that in the case of $R_{c}=$ const. as also seen for $R_{a}=1.0$ (Figs. 8 and 13). However, the local increase of $\Delta \theta_{y, i j}$ in the vicinity of $z^{*}=0.0$ by the increase of $\alpha$, which is observed for $R_{a}=1.0$, is not recognized for $R_{a}=3.0$. This is attributed to the weaker interaction between a pair of passage vortex for $R_{a}=3.0$ than for $R_{a}=1.0$. As a result, a pair of small vortex around $z^{*}=0.0$ on the inner wall, which appears for $R_{a}=1.0$ (Fig. 14), is not induced.

Figure 22 shows the influence of $\alpha$ on the loading coefficient $A_{c p s}$ for the duct with $R_{a}=3.0$. The increase of $A_{c p s}$ by the increase of $\alpha$ is almost the same for the cases of $L_{c}=$ const. and $R_{c}=$ const., though it is higher for the case of $L_{c}=$ const. in $R_{a}=1.0$ (Fig. 16).

Figures 23 and 24 show the influences of $\alpha$ on the $\Delta C_{p t, i}$ and the $C_{p t, i}$ at the exit of the bend $(\theta=\alpha)$ for the duct with $R_{a}=3.0$, respectively. Those at $X_{D}=2.5$ downstream of the bend are also given in Figs. 25 and 26, respectively. At the exit of the bend, as the same as those for the duct with $R_{a}=1.0$ (Figs. 17 and 18), the $\Delta C_{p t, i}$ is higher for the case of $L_{c}=$ const. due to the stronger pas- 


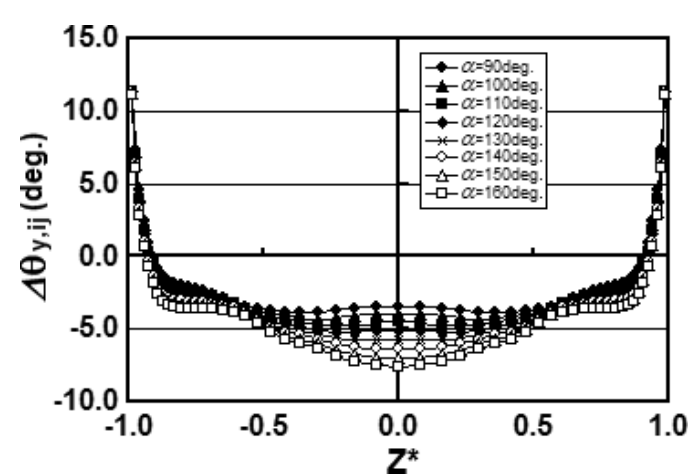

(a) $L_{c}=$ const.

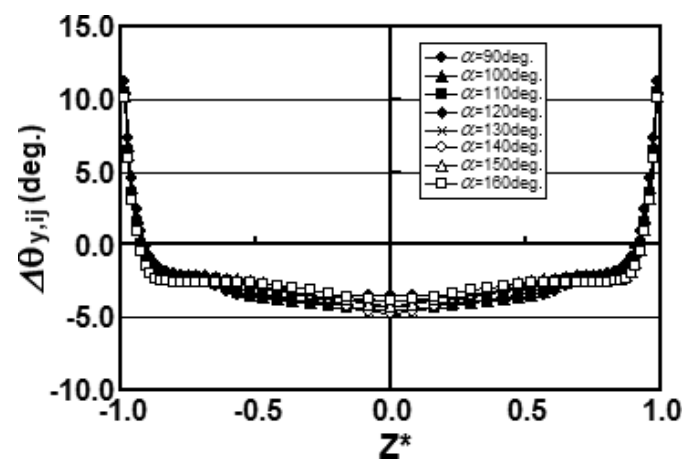

(b) $R_{c}=$ const.

Fig. 21 Flow angle at $\theta=\alpha\left(R_{a}=3.0\right)$

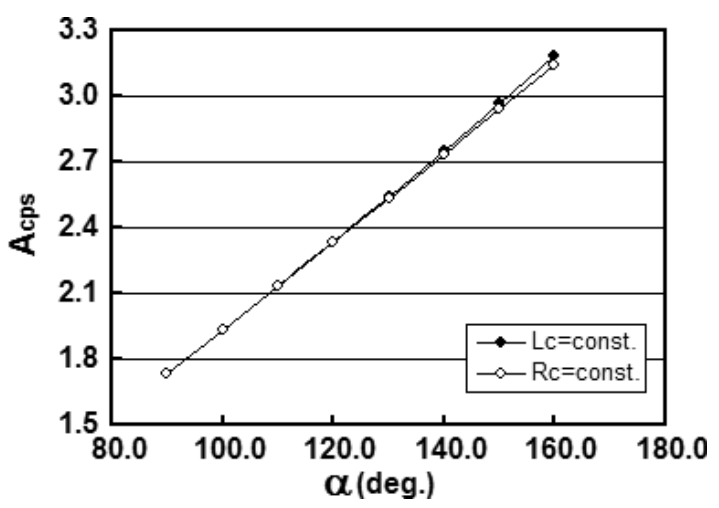

Fig. 22 Influence of $\alpha$ on loading coefficient $A_{c p s}\left(R_{a}=3.0\right)$

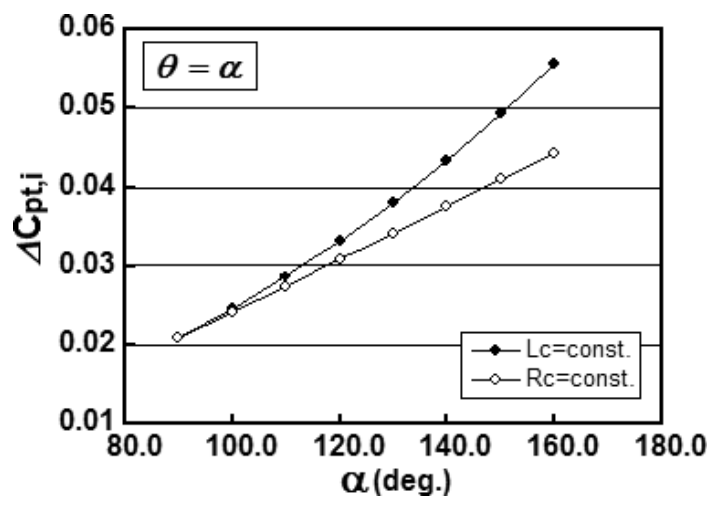

Fig. 23 Influence of $\alpha$ on $\Delta C_{p t, i}$ at $\theta=\alpha\left(R_{a}=3.0\right)$

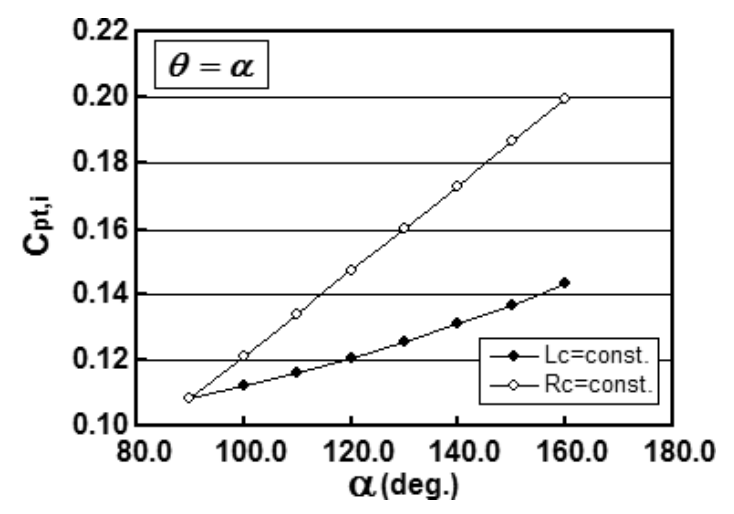

Fig. 24 Influence of $\alpha$ on $C_{p t, i}$ at $\theta=\alpha\left(R_{a}=3.0\right)$

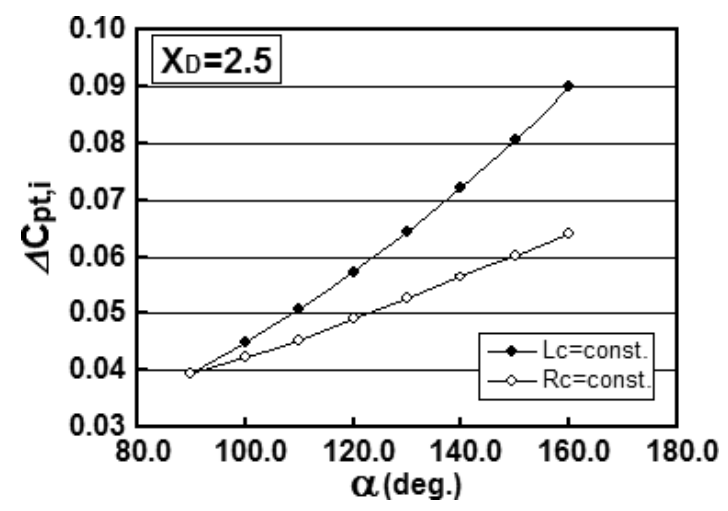

Fig. 25 Influence of $\alpha$ on $\Delta C_{p t, i}$ at $X_{D}=2.5\left(R_{a}=3.0\right)$

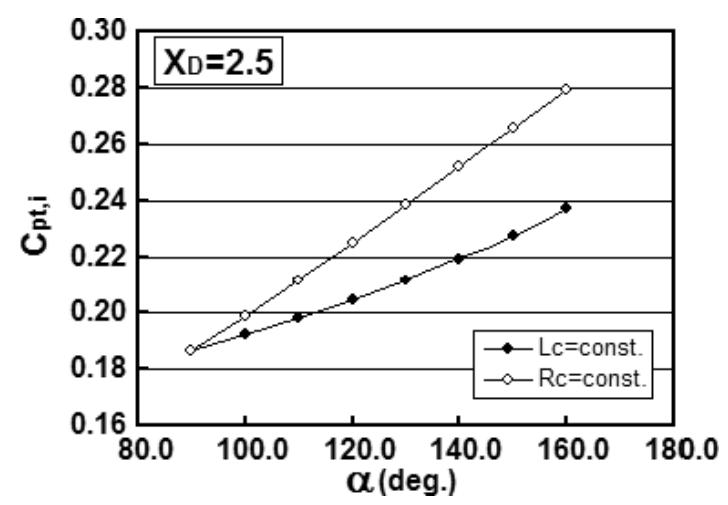

Fig. 26 Influence of $\alpha$ on $C_{p t, i}$ at $X_{D}=2.5\left(R_{a}=3.0\right)$

sage vortex in the case of $L_{c}=$ const. (Fig. 23), and the $C_{p t, i}$ becomes higher for the case of $R_{c}=$ const. due to the friction loss increased by the increase of $L_{c}$ (Fig. 24). Downstream of the bend, the $\Delta C_{p t, i}$ is higher for the case of $L_{c}=$ const. by the increase of $\alpha$ (Fig. 25) as also seen for $R_{a}=1.0$ (Fig. 19). However, the $C_{p t, i}$ becomes higher for the case of $R_{c}=$ const. as the $\alpha$ increases (Fig. 26). This phenomenon is different from that for $R_{a}=1.0$ (Fig. 20). As described in the results for $R_{a}=1.0$, the loss caused by the passage vortex becomes predominant in the overall loss generation downstream of the bend due to the decline of passage vortex developed within the bend. However, the development of the passage vortex within the bend for 
$R_{a}=3.0$ is weaker than that for $R_{a}=1.0^{(2)}$. Thus, as known by the comparison between Figs. 23 and 25, the increase of the $\Delta C_{p t, i}$ from the exit of the bend $(\theta=\alpha)$ up to $X_{D}=2.5$ is less than that for $R_{a}=1.0$ (Figs. 17 and 19), and consequently the $C_{p t, i}$ becomes lower for the case of $L_{c}=$ const. in the duct with $R_{a}=3.0$.

The results in the present study and in the second report $^{(2)}$ imply the followings. The increase of $\alpha$ more restrains the increase of the loss for the case of $R_{c}=$ const., but more increases the loading coefficient $A_{c p s}$ for the case of $L_{c}=$ const. in the duct with $R_{a}$ less than 2.0, which strongly generates the loss caused by the passage vortex. On the other hand, in the duct with $R_{a}$ higher than 2.0, the increase of $\alpha$ in the case of $L_{c}=$ const. is able to increase the $A_{c p s}$ in restraint of the increase of loss. These results in the stationary curved duct suggest that the increase of blade loading of an axial flow turbine by the increase of turning angle can be attained in restraint of the increase of loss by restraining the enlargement of blade surface area and by increasing the cross-sectional aspect ratio of the blade-to-blade channel to reduce the interaction between a pair of passage vortex.

\section{Conclusions}

The flows within curved ducts were analyzed by varying the curved angle with fixing the length or the mean radius of curvature of the bend. The following conclusions were derived from the present study.

(1) The increase of the curved angle with fixing the length of the bend increases the loss caused by the passage vortex which is strengthened by the increase of the difference between the static pressures on the inner and on the outer walls within the bend caused by the decrease of the mean radius of curvature of the bend. On the other hand, the increase of the curved angle with fixing the mean radius of curvature of the bend increases the friction loss due to the increase of the wall surface area.

(2) The increase of the curved angle with fixing the length of the bend decreases the flow angle at the exit of the bend by the increase of the strength of passage vortex. The fixed mean radius of curvature of the bend hardly influences it due to the almost constant strength of the passage vortex.

(3) For the duct with the low cross-sectional aspect ratio, in which the loss caused by the passage vortex is predominant within the overall loss, the increase of curved angle with fixing the mean radius of curvature of the bend more restrains the increase of loss. On the other hand, the fixed length of the bend more increases the difference between the static pressures on the inner and the outer walls.
(4) For the duct with the high cross-sectional aspect ratio, in which the loss caused by the passage vortex is minor within the overall loss, the increase of curved angle with fixing the length of the bend is able to increase the difference between the static pressures on the inner and the outer walls in restraint of the increase of loss.

( 5 ) The increase of blade loading of an axial flow turbine by the increase of the turning angle can be attained in restraint of the increase of loss by restraining the enlargement of blade surface area and by increasing the crosssectional aspect ratio of the blade-to-blade channel to reduce the interaction between a pair of passage vortex.

\section{References}

( 1 ) Tsujita, H., Ishida, Y., Mizuki, S. and Yamamoto, A., Investigation for Loss Generation Mechanisms of Flow in Turbomachinery by Using Curved Square Duct (1st Report, Influence of Inlet Velocity Distribution), Trans. Jpn. Soc. Mech. Eng., (in Japanese), Vol.69, No.683, B (2003), pp.1628-1636.

( 2 ) Tsujita, H., Mizuki, S. and Yamamoto, A., Investigation for Loss Generation Mechanisms of Flow in Turbomachinery by Using Curved Square Duct (2nd Report, Influence of Cross-Sectional Aspect Ratio), Trans. Jpn. Soc. Mech. Eng., (in Japanese), Vol.70, No.694, B (2004), pp.1496-1503.

( 3 ) Yamamoto, A. and Outa, E., Low-Speed Annular Cascade Tests of an Ultra-Highly Loaded Turbine with Tip Clearance, Part 1-Near Design Incidence, ASME Paper 99-GT-212, (1999).

(4) Yamamoto, A., Usui, H., Mizuki, S., Tsujita, H. and Outa, E., Research of Ultra-Highly Loaded Turbines, Proc. the 1st International Symposium of Environmentally Compatible Propulsion System for NextGeneration Supersonic Transport, C-9, (2002).

( 5 ) Tsujita, H., Mizuki, S. and Yamamoto, A., Numerical Analysis of Flow within Ultra-Highly Loaded Turbine Cascade, Trans. Jpn. Soc. Mech. Eng., (in Japanese), Vol.70, No.697, B (2004), pp.2332-2340.

(6) Tsujita, H. and Mizuki, S., Numerical Calculation of Incompressible Viscous Flow within Curved Duct Using Momentum Equations in Physical Component Tensor Form for Boundary-Fitted Coordinate System, Trans. Jpn. Soc. Mech. Eng., (in Japanese), Vol.57, No.540, B (1991), pp.2606-2613.

( 7 ) Tsujita, H. and Mizuki, S., Numerical Analysis of Compressible Flow Using Pressure-Correction Method, Trans. Jpn. Soc. Mech. Eng., (in Japanese), Vol.63, No.608, B (1997), pp.1270-1278.

( 8 ) Moore, J. and Adhye, R.Y., Secondary Flows and Losses Downstream of a Turbine Cascade, Trans. ASME, J. Eng. Power, Vol.107, No.10 (1985), pp.961968. 